\title{
Solar and Atmospheric influences in the Cosmic Ray Intensity
}

\author{
Rafael R. S. de Mendonça ${ }^{1}$ \\ National Institute For Space Research - INPE \\ E-mail: mendonca@dge.inpe.br
}

\section{Jean-Pierre Raulin}

Centro de Radio Astronomia e Astrofisica Mackenzie - CRAAM

\section{Fernando C. P. Bertoni}

Centro de Radio Astronomia e Astrofisica Mackenzie - CRAAM

\section{Ezequiel Echer}

National Institute For Space Research - INPE

\section{Vladimir S. Makhmutov}

P. N. Lebedev Physical Institute - LPI

\section{Germán Fernández}

Complejo Astronomico El Leoncito - CASLEO

Cosmic ray intensity at Earth changes with time and presents variations associated with the 11yr solar cycle, with interplanetary coronal mass ejections (ICME) arriving on the Earth's magnetosphere, and with energetic particles produced during large solar flares. Studies also show that the cosmic rays can be related with atmospheric phenomena. Since cosmic rays are the main source of ionization between $\sim 4$ and $\sim 60 \mathrm{~km}$, they may influence climate phenomena as global cloud coverage and rain precipitation rate. Meteorological and atmospheric processes, such as changes in the ambient pressure and temperature, rain precipitation and variations of the atmospheric electric field can affect the cosmic ray counts observed by ground based detectors. In this work we present the results obtained with the CARPET charged particle detector installed at Astronomical Complex El Leoncito (CASLEO) in San Juan - Argentina, since April 2006. We show and discuss the long term modulation of the cosmic ray flux associated with solar phenomena, like the 11-yr solar cycle and seasonal variations that can be related to changes in the atmospheric temperature. We also correct cosmic ray data for the barometric effect in order to discuss short time increases of the cosmic ray intensity in association to rain precipitation and changes in the atmospheric electric field.

4th School on Cosmic Rays and Astrophysics

Sao Paulo, Brazil

August 25- September 04, 2010

\footnotetext{
${ }^{1}$ Speaker
} 


\section{Introduction}

It is known that solar phenomena and, interplanetary medium and magnetospheric disturbances produce variations in the cosmic ray intensity observed at Earth's surface. The better known solar influence in the cosmic ray flux is the 11-yr variation. There is an anti-correlation between the 11-yr solar cycle and the cosmic ray intensity due to the inhomogeneity of the solar magnetic field during the solar maximum period. Further there is also an influence of the 22-yr solar magnetic field polarity inversion cycle [1]. Solar Flares can accelerate particles in the solar corona which can be detected as cosmic ray count increases using ground based detectors [2].

Other typical variations of the cosmic ray count rate observed on the Earth's surface are decreases known as Forbush events, which are due to the scattering of cosmic rays by shockwaves and/or the magnetic fields associated with magnetized masses ejected from the sun [3].

Recent studies have shown that cosmic ray intensity variations can also affect local and global processes in the Earth's atmosphere. It is possible that variations in the cosmic ray flux influence the global cloud coverage, the rain precipitation rate, the air ionization, the ionosphere, the radio wave propagation, the global electric circuit and the ozone layer formation $[4,5,6]$.

On the other hand, atmospheric processes and phenomena can in turn influence the cosmic ray intensity observed by ground detectors. Changes in the atmospheric pressure, temperature, the atmospheric electric field and rain water precipitation produce variations in cosmic ray count rate of ground-based detectors.

The influence of the atmospheric pressure in the cosmic ray intensity, also called the barometric effect, has been studied since the discovery of cosmic rays. Basically, the influence of the atmospheric pressure can be described as an absorber effect, the higher the atmospheric pressure, the higher the collision rate between cosmic rays and atmospheric particles at high altitudes, producing less cosmic ray counts at low altitudes. More precisely, the barometric effect produces an anti-correlation between the atmospheric pressure and the cosmic ray intensity [7].

The atmospheric temperature influence can be explained as a decrease of the muons intensity at ground level because of the increase of the propagation path of múons, and consequent increased probability of decay, which occurs due to the heating and expansion of the atmosphere [7].

Changes in the atmospheric electric field due to the presence of electrically charged clouds (thunderstorm clouds) can produce variations in the cosmic ray counts detected by ground detectors. Secondary cosmic rays (e.g. electrons and positrons) can be accelerated by the electric field of the thunderstorm clouds producing an increase of the detected count rate. Very strong increases in the atmospheric electric field during thunderstorms can also accelerate (or decelerate) muons and protons affecting the count rate of the high-energy muons and neutrons detectors. The type of variation of the cosmic ray count observed by a detector depends on which particle is detected and on its energy $[8,9,10]$. 
Using low-energy cosmic ray detectors, count increases can be observed during rain periods. The reason is that isotopes of ${ }^{222} \mathrm{Rn},{ }^{214} \mathrm{Bi}$ and ${ }^{214} \mathrm{~Pb}$ floating in the atmosphere in the form of free ions or aerosols can be transported down to the ground by rain droplets, causing an increase of the $\gamma$-rays detected at the ground level $[11,12]$.

With the purpose to study the variations of cosmic ray intensity related to atmospheric and solar phenomena, as well as caused by interplanetary modulation processes and solar flares, we installed in 2006, in San Juan (Argentine Andes) the CARPET cosmic ray detector.

\section{The CARPET cosmic ray detector}

The cosmic ray detector CARPET, showed in Fig. 1-A, was installed on April 2006 at the El Leoncito Astronomical Complex (CASLEO) in San Juan - Argentina at an altitude of $2552 \mathrm{~m}$ where the geomagnetic rigidity cutoff is $11.8 \mathrm{GV}$. It was developed and designed at the Lebedev Physical Institute (LPI) in Russia within an international scientific cooperation between LPI, the Center of Radio Astronomy and Astrophysics Mackenzie (CRAAM) from Brazil and the CASLEO.

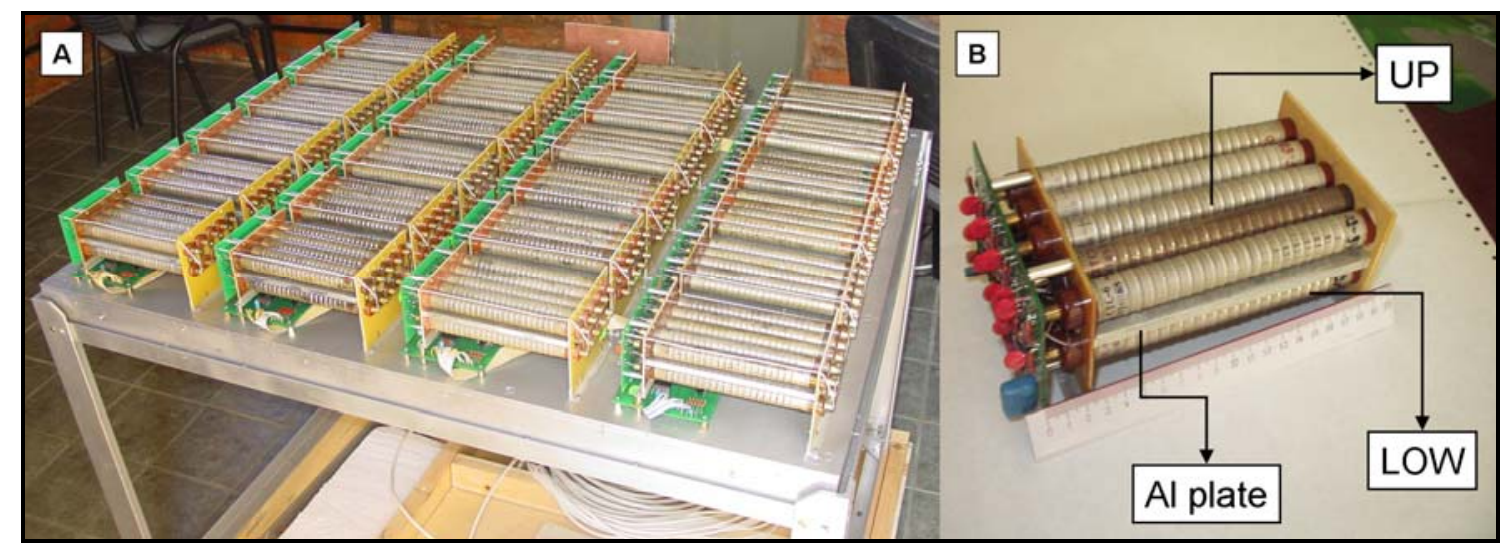

Figure 1: A - Inner vision of the CARPET detector. B - Detail of one block of the CARPET detector with 5 upper and 5 lower counters separated by an aluminum plate.

The CARPET consists of 24 blocks of 10 gas-discharge Geiger counters (STS-6) located on a platform of $\sim 1.5 \mathrm{~m} \mathrm{x} \sim 1.5 \mathrm{~m}$ (Fig. 1-A). Each counter has a diameter of $1.9 \mathrm{~cm}$ and a length of $9.8 \mathrm{~cm}$. As shown in Figure 1-B, each block consists of five upper and five lower counters separated by an aluminum absorber plate with a thickness of $7 \mathrm{~mm}$.

The data of this detector consist in three channels: UP and LOW, which measure the total count rate detected respectively by the 120 tubes above and below the Al plate; and TELESCOPE, which corresponds to the total count of particles crossing the aluminum plate and are detected simultaneously by one upper and one lower counter in the same block. The cutoff energy for the UP and LOW channels is: $\mathrm{E}>0.2 \mathrm{MeV}$ for electrons (e), E $>5.0 \mathrm{MeV}$ for protons $\left(\mathrm{p}^{+}\right)$and $\mathrm{E}>20 \mathrm{keV}$ for photons (detection efficiency $<1 \%$ ). For the TELECOPE we have: $\mathrm{E}>5 \mathrm{MeV}$ for $\mathrm{e}^{-}, \mathrm{E}>30 \mathrm{MeV}$ for $\mathrm{p}^{+}$and $\mathrm{E}>20 \mathrm{MeV}$ for muons $(\mu)$. The TELESCOPE channel is not sensitive to $\gamma$-rays such as UP and LOW channels. The UP and LOW channels are more sensitive to the electromagnetic (soft) component of the cosmic ray shower, while the TELESCOPE channel is more sensitive to the muonic (hard) component. 


\section{The barometric and temperature effects in the CARPET data}

In order to study the barometric effect in the cosmic ray count, we performed an analysis of the CARPET's data and the atmospheric pressure variations observed in July 2009. This period was chosen because there was no significant influence of other atmospheric, geomagnetic and solar phenomena. During this period, no rains were observed neither significant changes of temperature and atmospheric electric field. Similarly, no geomagnetic disturbances, no transient solar events such as flares and mass ejections occurred during this period.

We analyzed the atmospheric pressure deviation $(\Delta \mathrm{P})$ and the relative deviation of the cosmic ray intensity $(\Delta \mathrm{I} / \mathrm{I})$ from the mean intensity. Figure 2 shows the correlation between these two parameters.

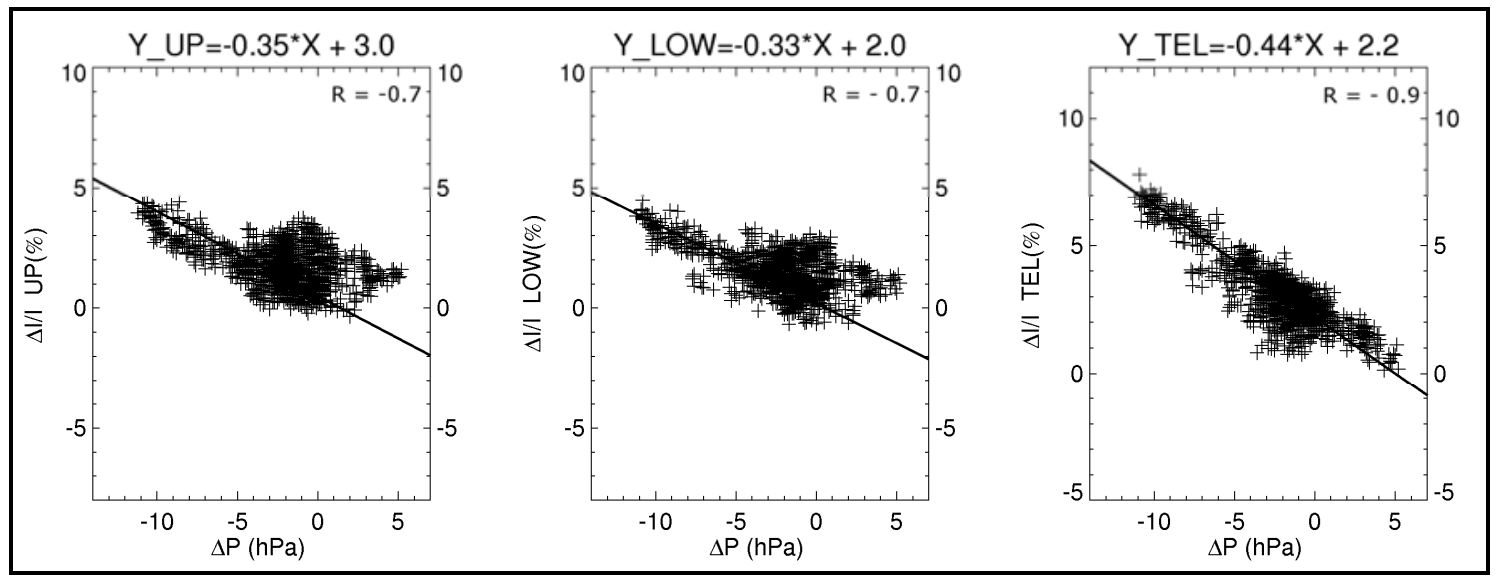

Figure 2: The anti-correlation between the pressure deviation and the relative variation of cosmic ray count for the UP, LOW and TELESCOPE channel.

The correlation coefficient $(\beta)$ we found is $\beta=-0.35 \% / \mathrm{hPa}$ for UP, $\beta=-0.33 \% / \mathrm{hPa}$ for LOW and $-0.44 \% / \mathrm{hPa}$ for TELESCOPE. Therefore, using these coefficients, it is possible to eliminate the pressure influence in the cosmic ray intensity observed with the CARPET detector.

In Figure 3 we note a clear seasonal wave (dotted red curve in the top panel) modulating the cosmic ray count rate, with maxima and minima during southern hemisphere winter and summer times, respectively. Analyzing the behavior of the surface temperature (green curve in bottom panel), it is suggested that the seasonal variation of the cosmic ray intensity is related to the temperature variations.

The atmospheric temperature influence was studied by the analysis of the temperature deviation $(\Delta \mathrm{T})$ and of the relative deviation of the cosmic ray intensity $(\Delta \mathrm{I} / \mathrm{I})$ observed between April 2006 and June 2010. Figure 4 shows the anti-correlation between these two parameters. 


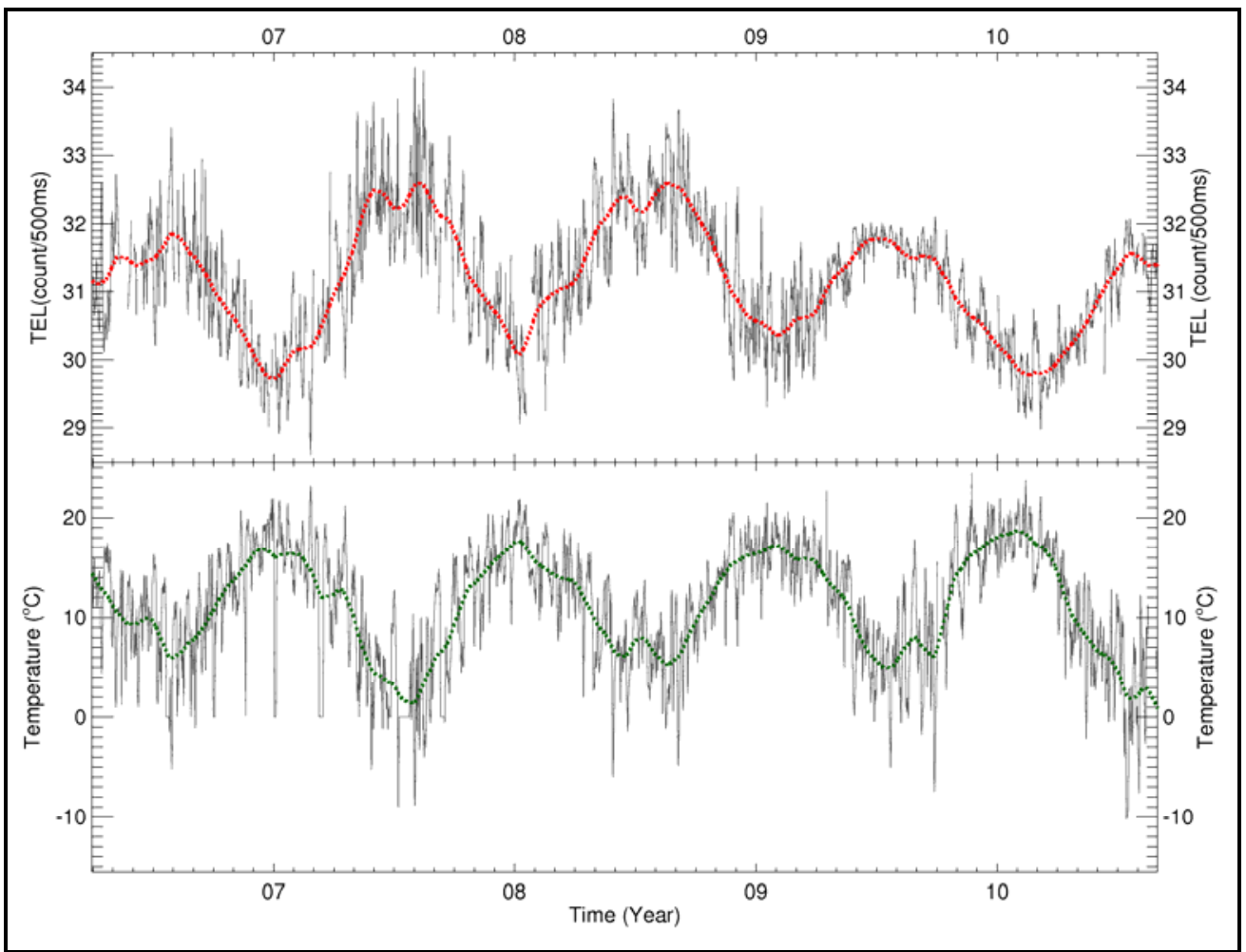

Figure 3: Top Panel - The cosmic ray intensity regarded by the TELESCOPE channel between April 2006 and September 2010 in different integration times: 24 hours (grey curve) and 15 days (red dotted curve). Bottom Panel - temperature variation observed during this period with 24 hours (grey curve) and 15 days (green dotted curve) integration times.

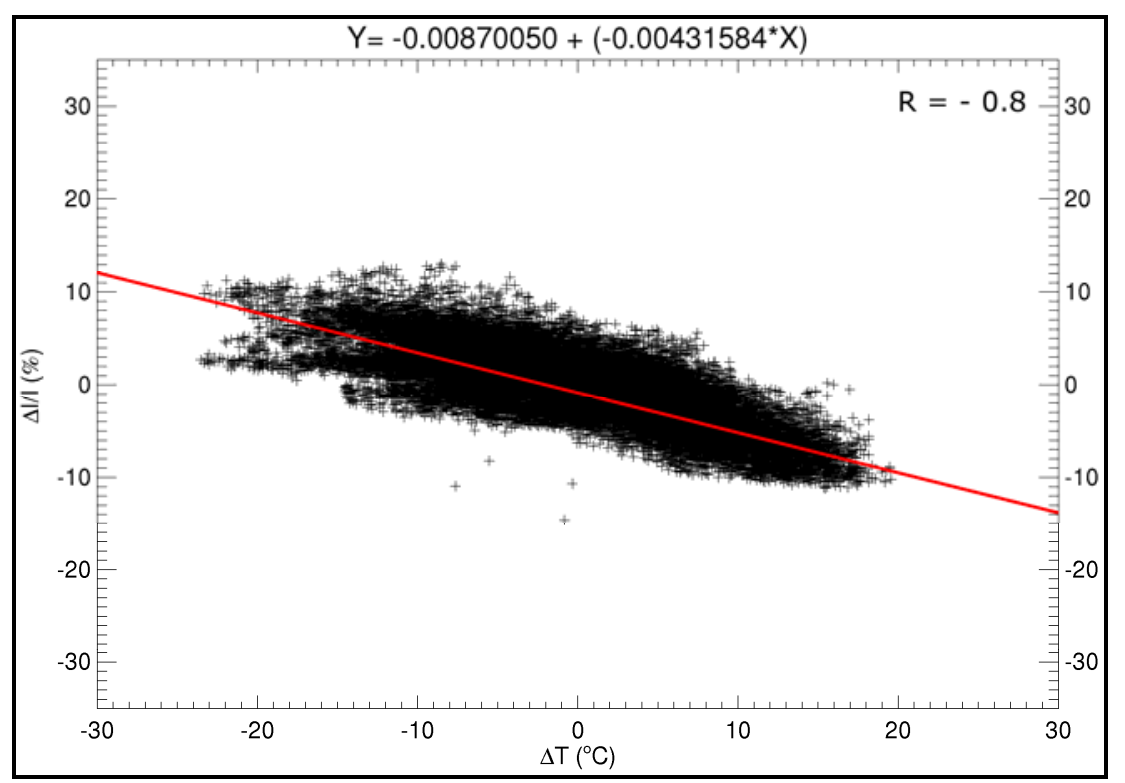

Figure 4: The anti-correlation between the temperature and the relative variation of cosmic rays count observed by the TELESCOPE channel.

\section{Solar influence in the cosmic ray flux}

Most of the operation of the CARPET detector occurred during descending phase and minimum epoch of solar cycle i.e. under low solar activity conditions. Certainly for this reason 
we did not detect any Ground Level Enhancement (GLE) events as it may be observed following large solar flares, nor did CARPET observed large Forbush decreases. Only few and faint decreases of the cosmic ray count rate $(\sim$ up to $5 \%)$ were identified during geomagnetic disturbances.

Since April 2006, only two solar flares were reported with a significant flux of ultraenergetic particles. Unfortunately, the CARPET detector was on a routine maintenance during the large solar flare on December $13^{\text {th }}, 2006$. The other event was smaller and occurred in December $6^{\text {th }}, 2006$. For this event, the CARPET observed a small increase in cosmic ray counts simultaneously in the three channels.

On a longer timescale, it is possible to identify in the CARPET data the influence of the solar activity cycle. In Figure 5 we compare the cosmic ray count rate observed by the TELESCOPE channel (bottom curves) with indices of the solar activity as the solar sunspot number and the $\mathrm{F} 10.7 \mathrm{~cm}$ radio flux (top curves). We also filtered the periodic variations in the TELESCOPE time profile, in order to remove the effect of surface temperature changes, as explained in the previous section. The result of this procedure is shown as a blue dashed line (bottom plot) which shows an increase between April 2006 and mid-2008, and a subsequent decrease until August 2010. The cosmic ray intensity maximum was occurred in the middle of 2008. The behavior of the smoothed TELESCOPE curve is then opposite to that of the solar activity, since the latter was decreasing until mid-2008 and then it increased toward the new cycle number 24 .

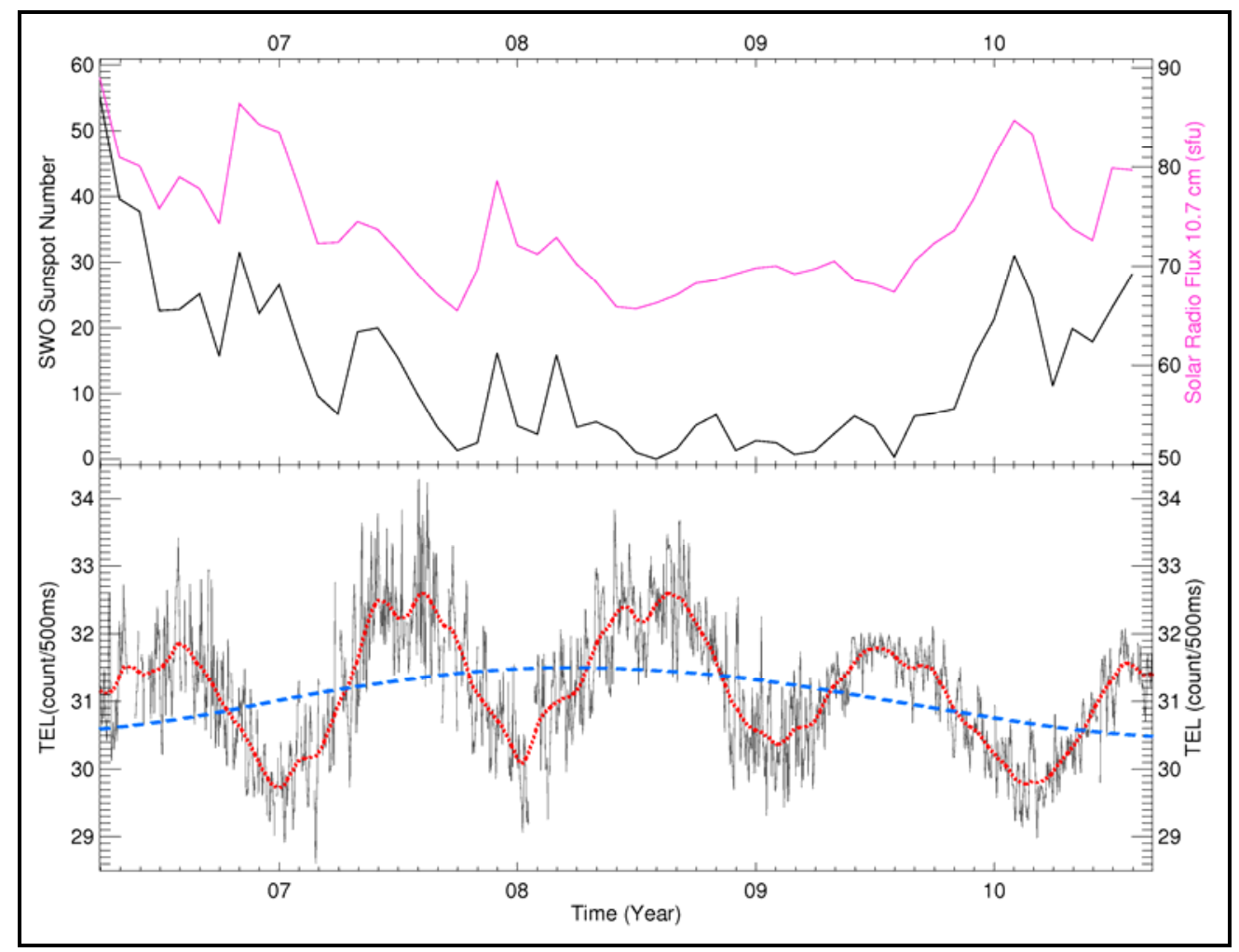

Figure 5: Upper panel: monthly means of Solar Radio Flux F10.7 (pink curve) and Sunspot number (black curve). Bottom Panel: Cosmic Rays variation as observed by TEL channel of the CARPET detector from April 2006 to September 2010 in different integration times: 24 hours (grey curve), 15 days (red dotted curve) and 6 months (blue dashed curve). 


\section{Influences of rain and atmospheric electric field (REF events)}

Several of the cosmic ray count increases detected by CARPET in the UP and LOW channels were observed during periods of thunderstorm activity, changes in the atmospheric electric field accompanied (or not) by rain precipitation. As we mentioned earlier, both of these processes might have contributed to the observed events by CARPET. In the following we will call REF events those events detected by CARPET for which both Rain precipitation and variations of the atmospheric Electric Field were observed. In Figure 6, we illustrate some examples of REF events observed in May $3^{\text {th }}, 2008$. For the whole period between April 2006 and July 2010, 135 REF events were observed.

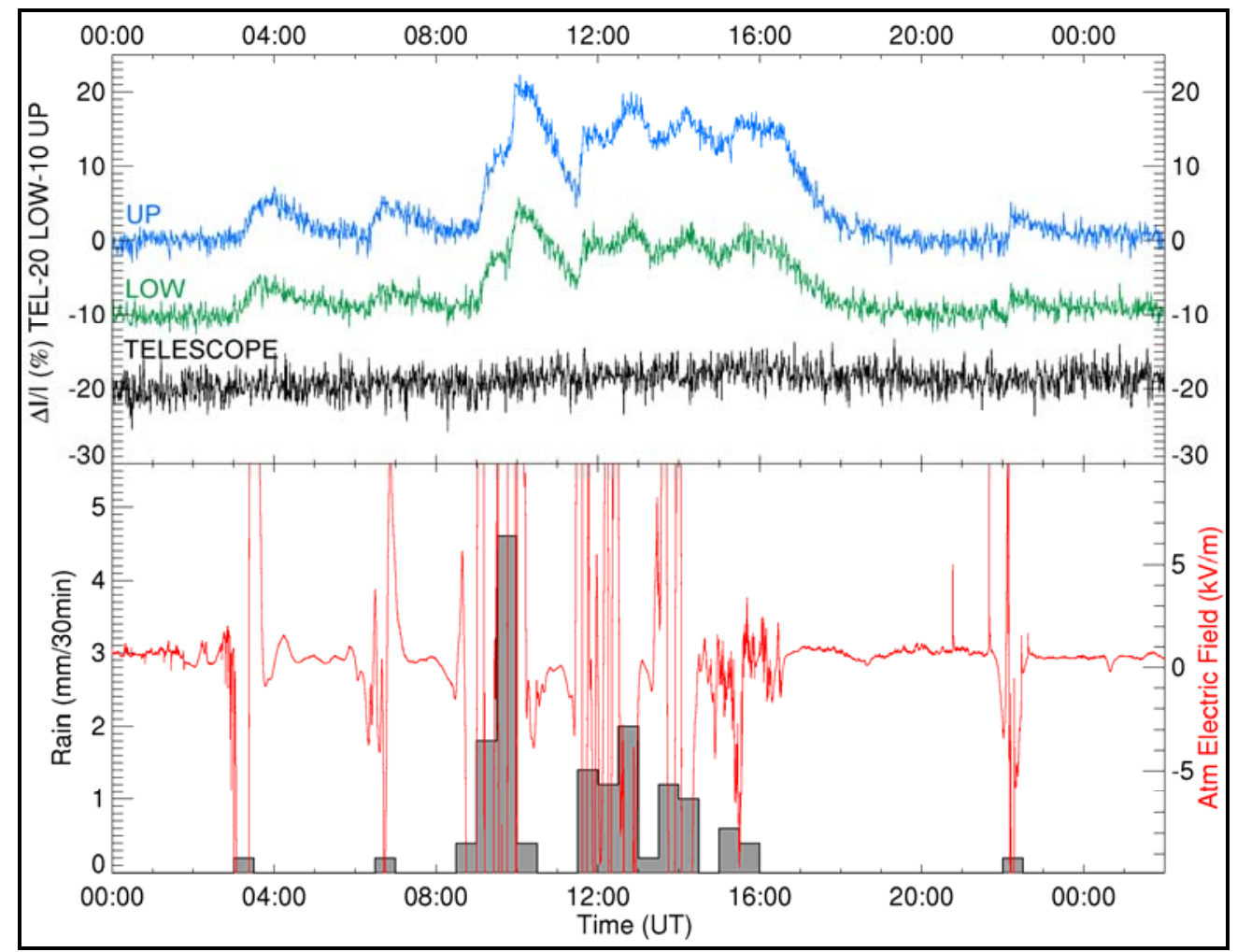

Figure 6: Example of increases in the cosmic ray count rate regarded by UP and LOW channels related to rain water precipitation (grey histogram) and to the atmospheric electric field (red curve). The plot period starts on 2008/03/10 at 00 UT and ends on 2008/03/11 at 02:00 UT.

\subsection{Analysis of geomagnetic activity, temperature and pressure variations during REF events}

In order to study the REF events it is necessary to investigate the behavior of other parameters which can also be associated with variations in cosmic ray intensity. Disturbances in the Earth's magnetic field modify the trajectory of cosmic rays and may change the geomagnetic rigidity at a given location, causing variations in the cosmic ray intensity at the ground-level [13]. As discussed previously, changes in the atmospheric pressure and temperature also produce variations in the cosmic ray count rate. Thus, we have checked for all REF events, the possible influence of the geomagnetic activity, temperature and pressure variations observed during the same period. 
The Kp and Dst indices are related to changes in the Earth's magnetic field which can be associated to geomagnetic storms and/or other related solar-interplanetary phenomena [14, 15]. Only $4.3 \%$ of REF events present $\mathrm{Kp} \geq 5$. According to the Dst index criteria of geomagnetic storm [16], the distribution of the Dst index values observed during the period of REF events is summarized in the Pie Chart 1 shown in Figure 7. This pie chart plot indicates that the large majority of REF events did occur during quiescent geomagnetic periods, with no storms, suggesting that they are not related to disturbances in the Earth's magnetic field.

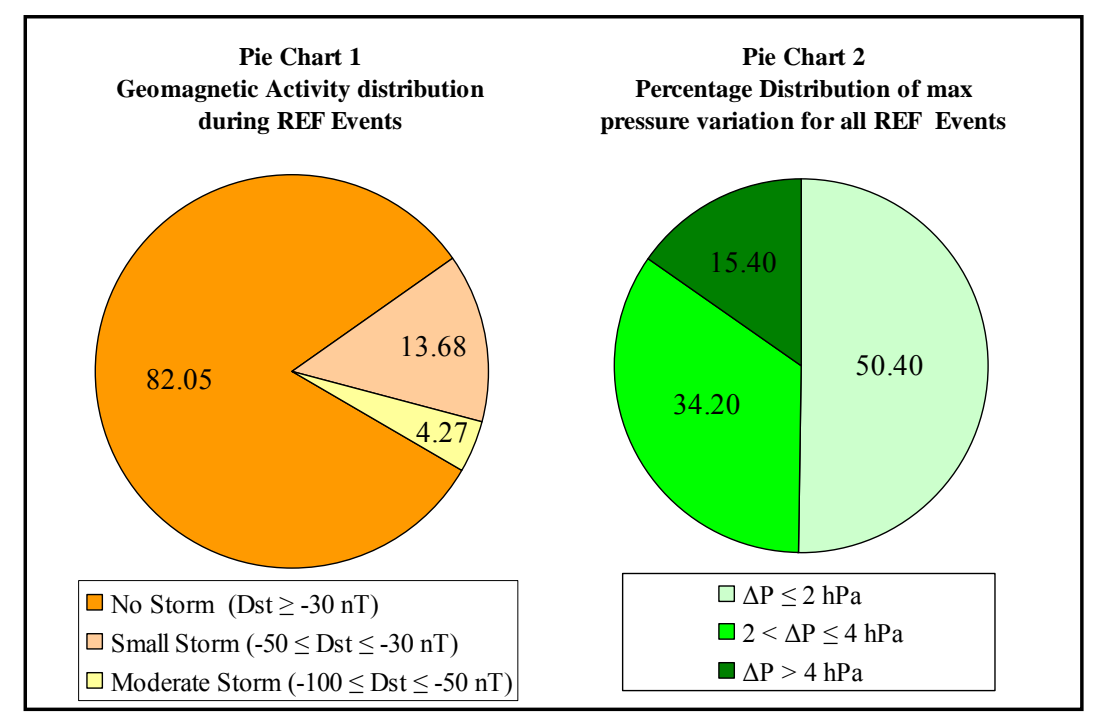

Figure 7: Percentage distribution of the geomagnetic storms during the REF events and the percentage distribution of the maximum pressure variation occurred during the REF events.

Although the CARPET data are corrected for the barometric effects, we analyzed the pressure variations during REF events in order to confirm that they could not have produced the cosmic ray intensity increases during periods of rain precipitation and rapidly varying atmospheric electric field. The maximum pressure variation observed during a REF event $(\delta \mathrm{P})$ was $9.8 \mathrm{hPa}$ and the mean value taking account all the REF events was $2.6 \pm 2.0 \mathrm{hPa}$. According to the barometric coefficient calculated for UP channel, $\delta \mathrm{P}$ and $\delta \mathrm{P}_{\mathrm{M}}$ would produce cosmic ray count variations of $3.4 \%$ and $0.9 \pm 0.7 \%$ respectively, while the variations observed during REF events are in the range $10-20 \%$. The distribution of $\delta \mathrm{P}$ values for all the REF events is shown in Figure 7, and it is clear that pressure variations are too small to account for REF events.

The maximum temperature variation observed during a $\mathrm{REF}$ event is $15.2{ }^{\circ} \mathrm{C}$, and its average for all the REF events is $3.0 \pm 2.6^{\circ} \mathrm{C}$. In most of the cases $(88 \%)$ the maximum temperature variation is $\leq 5{ }^{\circ} \mathrm{C}$, which is generally smaller than the variations observed during periods without any REF events at all. Therefore, most likely, temperature variations had no influences on the occurrence of the observed REF events.

\subsection{Study about the rain precipitation influence in the REF Events}

Among a total of 135 REF events detected, we analyzed 64 events which were classified as simple events. A simple event is a rise and fall of the cosmic ray flux, isolated in time, like the examples shown in Figure 6 at $\sim 03: 00, \sim 06: 30$ and $\sim 22: 00$ UT. In contrast, complex events 
are composed of superimposed rises and falls, not resolved in time, like during the time interval 08:30 - 16:00 in Figure 6. For all the simple REF events, we calculated a parameter called the impulsivity, which corresponds to the time integrated count rate curve between the starting time and the peak time. We also measured the accumulated precipitated rain during the same period. The result of this analysis is shown in Figure 8 where we correlated both of the above mentioned quantities.

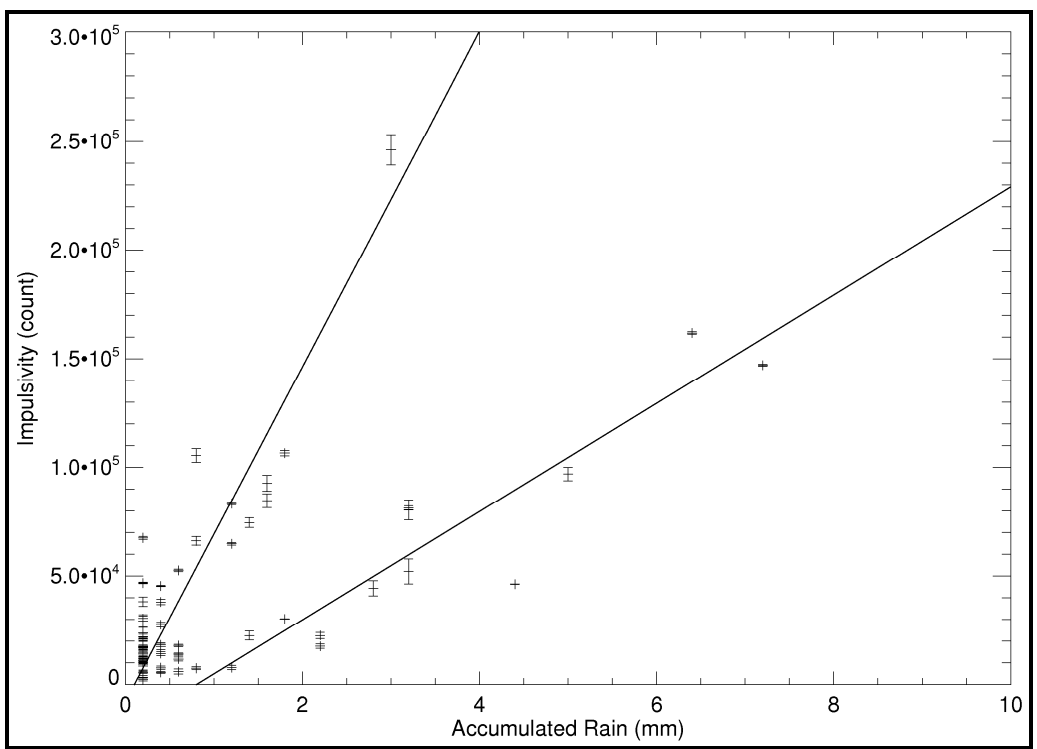

Figure 8: Correlations of the impulsivity and the accumulated rain obtained through an analysis of 64 simple events recorded between April 2006 and January 2010.

We found two distinct linear correlations, suggesting that other processes beyond the rain precipitation may generate increases in cosmic ray count recorded by the CARPET detector.

\subsection{Influence of the atmospheric electric field in the REF events}

As already mentioned earlier, atmospheric electric field variations can produce an increase in the cosmic ray flux. In the case of the CARPET detector, atmospheric electric field changes may produce cosmic ray count variations in the UP and LOW signals, and, sometimes, during large variations, count rate variations are observed in the TELESCOPE signal too. In Figure 9 we show an example of the REF event with a small variation in the TELESCOPE channel count rate which may have been triggered by a change in the atmospheric electric field during the same period. Beside the REF events, CARPET has also detected cosmic ray count rate increases during which no rain precipitation at all was observed, but only changes in the atmospheric electric field [17]. These events, along with the two correlation lines shown in Figure 8, strongly suggest that electric field variations in the Earth's atmosphere may have a prime role in the cosmic ray increasing rate events observed by CARPET. A quick and simple interpretation would be that one the correlations in Figure 8 traduces the action of rain precipitation along with large atmospheric electric field variations, while the other may have been caused by rain precipitation along with moderate electric field variations. 


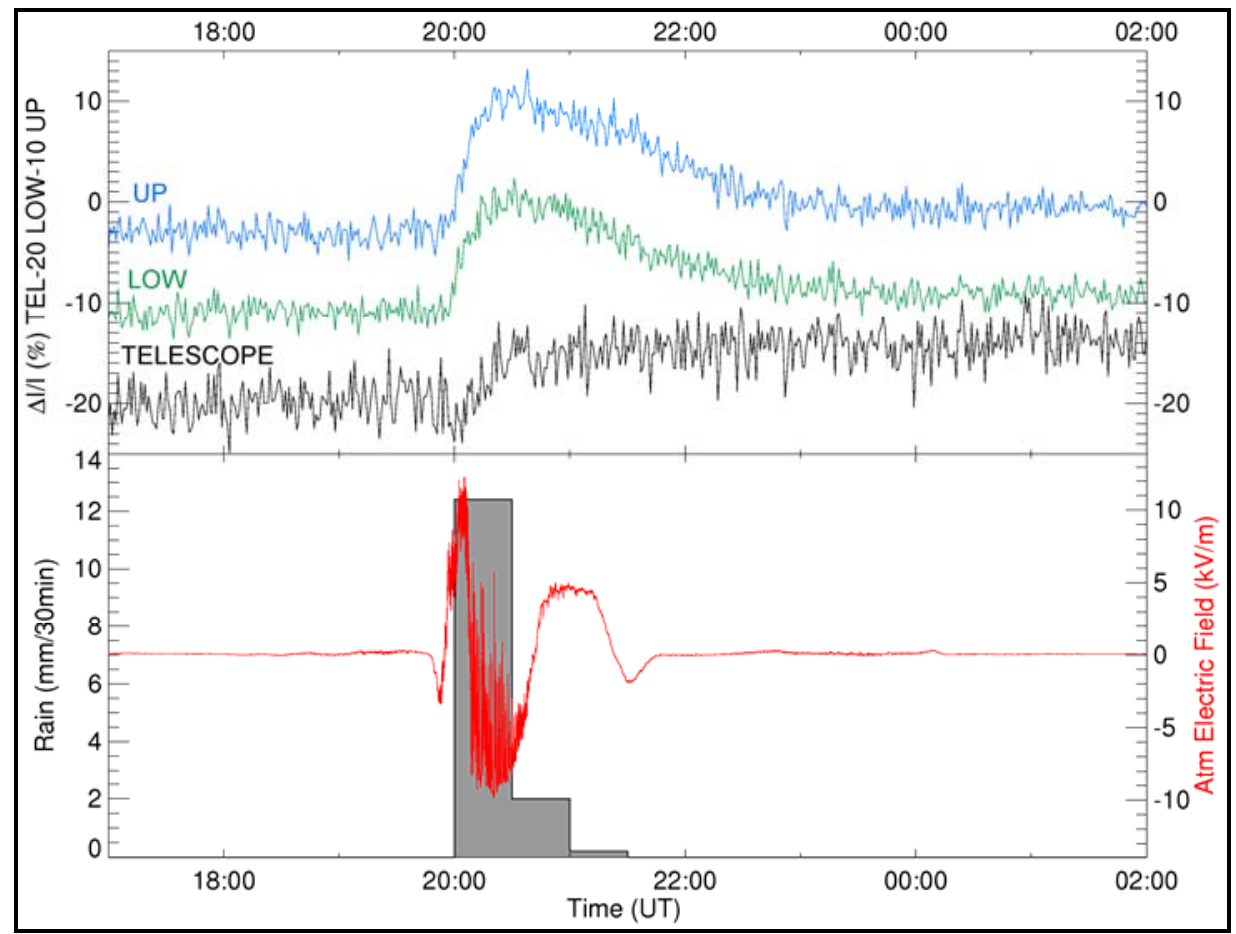

Figure 9: Variation in TELESCOPE count rate (black curve) during a period of rain precipitation (grey histogram) and atmospheric electric field (red curve) variation. The data range is 2010/01/23 at 17 UT to 2010/01/24 at 02 UT.

\section{Conclusion}

In this work we have presented observations obtained with the CARPET instrument installed at El Leoncito (CASLEO, San Juan, Argentina) since April 2006. Cosmic ray count rates were corrected for the barometric effect.

Increases of the cosmic ray count rate, called REF events, were detected during periods of rain precipitation and atmospheric electric field variations. We showed that the geomagnetic activity, as well as pressure and temperature variations, have no influence in the occurrence of REF events. The relation between REF event characteristics and the amount of rain precipitation during these events shows two clear correlations, indicating probably that the atmospheric electric field variations play certainly an important role in the occurrence of REF events.

We have also shown that the cosmic ray intensity measured by the high-energy channel of the CARPET presents a clear anti-correlation with the solar activity cycle. The cosmic ray intensity maximum at El Leoncito position was occurred in the middle of 2008. Seasonal variations in the cosmic ray intensity, with pronounced maxima during local winter times, were successfully compared with the surface temperature. However, to better understand this relation, a comparison with detailed height temperature profile is needed. Further works also include the study of the relation between REF events, the variations observed in the atmospheric electric field, and the occurrence of lightning discharges. 


\section{References}

[1] H. Moraal, Observations of 11-Year Cosmic-Ray Modulation Cycle, Space Sci. Rev. 19, 8451976.

[2] L. I. Miroshnichenko, Solar Cosmic Rays, Kluwer Academic Publishers, Dordrecht, 2001.

[3] H. V. Cane, Coronal mass ejections and Forbush decreases, Space Sci. Rev. 93, 55, 2000.

[4] G. A Bazilevskaya; M. B. Krainev; V. S. Makhmutov, Effects of cosmic rays on the Earth's environment, J. Atmos. Sol. Terr. Phys. 62, 1577, 2000.

[5] Y. I. Stozhkov; N. S. Svirzevskii; V. S. Makhmutov, Cosmic ray measurements in the atmosphere, in proceedings of Workshop on Ion-Aerosol-Cloud Interactions, Geneva, 41, 2001.

[6] H. Svensmark, Cosmic rays and Earth's climate, Space Sci. Rev. 93, 175, 2000.

[7] L. I. Dorman, Cosmic Rays in the Earth's Atmosphere and Underground, Kluwer Academic Publishers, Dordrecht, 2004.

[8] A. S. Lidvansky, The effect of the electric field of the atmosphere on cosmic rays, J. Phys. G: Nucl. Part. Phys. 29, 925, 2003.

[9] L. I. Dorman et al, Thunderstorms' atmospheric electric field effects in the intensity of cosmic ray muons and in neutron monitor data, J. Geophys. Res. Space Phys. 108(A5), 1181, 2003.

[10] V. V. Alexeenko et al, Transient variations of secondary cosmic rays due to atmospheric electric field and evidence for pre-lightning particle acceleration, Phys. Lett. A 301 (3-4), 299, 2002.

[11] A. Katase et al, Variation of Intensity Environmental Gamma-Rays Measured with Ge(Li) Spectrometer, J. Nucl. Sci. Tech. 19(11), 56, 1982.

[12] T. Ichiji; T. Hattori, Continuous measurement of environmental gamma-ray radiation in Tokyo using Ge semiconductor detector, in proceedings of the IRPA $11^{\text {th }}$ Conference, Madrid, 2004.

[13] L. I. Dorman, Cosmic Rays in Magnetosphere of the Earth and other Planets, Springer, Netherlands, 2009.

[14] G. Rostoker, Geomagnetic indices, Rev. Geophys., 10(4), 935, 1972.

[15] M. Sugiura; Hourly values of equatorial Dst for IGY, in Annals of the International Geophysical Year, Vol. 35, Pergamon Press, Oxford, 1964.

[16] W. D. Gonzalez et al, What is a geomagnetic storm, J. Geophys. Res. Space Phys. 99(A4), 5771, 1994.

[17] R. R. S. de Mendonça et al, Observation of Cosmic Ray and Electric Field Variations in the Surface Atmosphere, Bull. Russ. Acad. Sci. Phys. 73, 404, 2009. 